\title{
Squares in products in arithmetic progression with at most one term omitted and common difference a prime power
}

\author{
by \\ Shanta Laishram (Waterloo, ON), \\ T. N. Shorey (Mumbai) and Szabolcs Tengely (Debrecen)
}

1. Introduction. For an integer $x>1$, we denote by $P(x)$ and $\omega(x)$ the greatest prime factor of $x$ and the number of distinct prime divisors of $x$, respectively. Further, we put $P(1)=1$ and $\omega(1)=0$. Let $p_{i}$ be the $i$ th prime number. Let $k \geq 4, t \geq k-2$ and $\gamma_{1}<\cdots<\gamma_{t}$ be integers with $0 \leq \gamma_{i}<k$ for $1 \leq i \leq t$. Thus $t \in\{k, k-1, k-2\}, \gamma_{t} \geq k-3$ and $\gamma_{i}=i-1$ for $1 \leq i \leq t$ if $t=k$. We put $\psi=k-t$. Let $b$ be a positive squarefree integer and we shall assume, unless otherwise specified, that $P(b) \leq k$. We consider the equation

$$
\Delta=\Delta(n, d, k)=\left(n+\gamma_{1} d\right) \cdots\left(n+\gamma_{t} d\right)=b y^{2}
$$

in positive integers $n, d, k, b, y, t$. It has been proved (see [SaSh03] and [MuSh04]) that (1.1) with $\psi=1, k \geq 9, d \nmid n, P(b)<k$ and $\omega(d)=1$ does not hold. Further, it has been shown in [TSH06] that the assertion continues to be valid for $6 \leq k \leq 8$ provided $b=1$. We show

Theorem 1. Let $\psi=1, k \geq 7$ and $d \nmid n$. Then (1.1) with $\omega(d)=1$ does not hold.

Thus the assumption $P(b)<k$ and $k \geq 9$ (in [SaSh03] and [MuSh04]) has been relaxed to $P(b) \leq k$ and $k \geq 7$, respectively, in Theorem 1 . As an immediate consequence of Theorem 1 , we see that (1.1) with $\psi=0, k \geq 7$, $d \nmid n, P(b) \leq p_{\pi(k)+1}$ and $\omega(d)=1$ is not possible. If $k \geq 11$, we relax the assumption $P(b) \leq p_{\pi(k)+1}$ to $P(b) \leq p_{\pi(k)+2}$ in the next result.

2000 Mathematics Subject Classification: Primary 11D61.

Key words and phrases: diophantine equations, arithmetic progressions, Legendre symbol, Chabauty method.

Research of Sz. Tengely was supported in part by the Magyary Zoltán Higher Educational Public Foundation. 
Theorem 2. Let $\psi=0, k \geq 11$ and $d \nmid n$. Assume that $P(b) \leq p_{\pi(k)+2}$. Then (1.1) with $\omega(d)=1$ does not hold.

For related results on (1.1), we refer to [LaSh08].

2. Notations and preliminaries. We assume (1.1) with $\operatorname{gcd}(n, d)=1$ in this section. Then we have

$$
n+\gamma_{i} d=a_{\gamma_{i}} x_{\gamma_{i}}^{2} \quad \text { for } 1 \leq i \leq t
$$

with $a_{\gamma_{i}}$ squarefree such that $P\left(a_{\gamma_{i}}\right) \leq \max (k-1, P(b))$. Thus (1.1) with $b$ as the squarefree part of $a_{\gamma_{1}} \cdots a_{\gamma_{t}}$ is determined by the $t$-tuple $\left(a_{\gamma_{1}}, \ldots, a_{\gamma_{t}}\right)$. Further, we write

$$
b_{i}=a_{\gamma_{i}}, \quad y_{i}=x_{\gamma_{i}} .
$$

Since $\operatorname{gcd}(n, d)=1$, we see from (2.1) that

$$
\left(b_{i}, d\right)=\left(y_{i}, d\right)=1 \quad \text { for } 1 \leq i \leq t .
$$

Let

$$
R=\left\{b_{i}: 1 \leq i \leq t\right\} .
$$

Lemma 2.1 ([LaSh08]). Equation (1.1) with $\omega(d)=1$ and $k \geq 9$ implies that $t-|R| \leq 1$.

Lemma 2.2. Let $\psi=0, k \geq 4$ and $d \nmid n$. Then (1.1) with $\omega(d)=1$ implies $(n, d, k, b)=(75,23,4,6)$.

This is proved in [SaSh03] and [MuSh03] unless $k=5, P(b)=5$, and then it is a particular case of a result of Tengely [Sz08].

Lemma 2.3 ([SaSh03, Theorem 4] and [MuSh04]). Let $\psi=1, k \geq 9$ and $d \nmid n$. Assume that $P(b)<k$. Then (1.1) with $\omega(d)=1$ does not hold.

Lemma 2.4 ([LaSh08]). Let $\psi=2, k \geq 15$ and $d \nmid n$. Then (1.1) with $\omega(d)=1$ does not hold.

Lemma 2.5. Let $\psi=1, k=7$ and $d \nmid n$. Assume that (1.1) holds. Then $\left(a_{0}, a_{1}, \ldots, a_{6}\right)$ is different from the following tuples and their mirror images:

$$
\begin{aligned}
& (1,2,3, *, 5,6,7),(2,1,6, *, 10,3,14),(2,1,14,3,10, *, 6), \\
& (*, 3,1,5,6,7,2),(3,1,5,6,7,2, *),(3, *, 5,6,7,2,1), \\
& (1,5,6,7,2, *, 10),(*, 5,6,7,2,1,10),(5,6,7,2,1,10, *), \\
& (6,7,2,1,10, *, 3),(10,3,14,1,2,5, *), \\
& (*, 10,3,14,1,2,5),(5,2,1,14,3,10, *),(*, 5,2,1,14,3,10) .
\end{aligned}
$$

Further, $\left(a_{1}, \ldots, a_{6}\right)$ is different from $(1,2,3, *, 5,6),(2,1,6, *, 10,3)$ and their mirror images.

The proof of Lemma 2.5 is given in Section 3.

The following result is contained in [BBGH06, Lemma 4.1]. 
LEMMA 2.6. There are no coprime positive integers $n^{\prime}, d^{\prime}$ satisfying the diophantine equations

$$
\begin{array}{ll}
\prod(0,1,2,3)=b y^{2}, & b \in\{1,2,3,5,15\}, \\
\prod(0,1,3,4)=b y^{2}, & b \in\{1,2,3,6,30\},
\end{array}
$$

where $\prod(0, i, j, l)=n^{\prime}\left(n^{\prime}+i d^{\prime}\right)\left(n^{\prime}+j d^{\prime}\right)\left(n^{\prime}+l d^{\prime}\right)$.

Lemma 2.7. Equation (1.1) with $\psi=1, k=7$ is not possible if

(i) $a_{1}=a_{4}=1, a_{6}=6$ and either $a_{3}=3$ or $a_{2}=2$,

(ii) $a_{1}=a_{6}=1$ and at least two of $a_{2}=2, a_{4}=6, a_{5}=5$ hold,

(iii) $a_{0}=a_{6}=2, a_{5}=3$ and either $a_{2}=6$ or $a_{4}=1$,

(iv) $a_{0}=a_{5}=1$ and at least two of $a_{1}=5, a_{2}=6, a_{4}=2$ hold,

(v) $a_{3}=a_{6}=1, a_{1}=6$ and $a_{2}=5$,

(vi) $a_{0}=a_{4}=1, a_{3}=3$ and $a_{6}=2$,

(vii) $a_{0}=a_{5}=1$ and at least two of $a_{1}=2, a_{3}=6, a_{6}=3$ hold.

Proof. The proof of Lemma 2.7 uses MAGMA to compute integral points on quartic curves. For this we first make a quartic curve and find an integral point on it. Then we compute all integral points on the curve by using the MAGMA command IntegralQuarticPoints and we exclude them.

We illustrate this with an example. Consider (ii). Then from $x_{6}^{2}-x_{1}^{2}=$ $n+6 d-(n+d)=5 d$ and $\operatorname{gcd}\left(x_{6}-x_{1}, x_{6}+x_{1}\right)=1$, we get either

$$
x_{6}-x_{1}=5, \quad x_{6}+x_{1}=d
$$

or

$$
x_{6}-x_{1}=1, \quad x_{6}+x_{1}=5 d .
$$

Assume (2.4). Then $d=2 x_{1}+5$. This with $n+d=x_{1}^{2}$ gives

$$
\begin{array}{ll}
2 x_{2}^{2}=n+2 d=n+d+d=x_{1}^{2}+2 x_{1}+5=\left(x_{1}+1\right)^{2}+4 & \text { if } a_{2}=2, \\
6 x_{4}^{2}=n+4 d=n+d+3 d=x_{1}^{2}+6 x_{1}+15=\left(x_{1}+3\right)^{2}+6 & \text { if } a_{4}=6, \\
5 x_{5}^{2}=n+5 d=n+d+4 d=x_{1}^{2}+8 x_{1}+20=\left(x_{1}+4\right)^{2}+4 & \text { if } a_{5}=5 .
\end{array}
$$

When $a_{2}=2, a_{4}=6$, by putting $X=x_{1}+1, Y=6 x_{2} x_{4}$, we get the quartic curve $Y^{2}=3\left(X^{2}+4\right)\left((X+2)^{2}+6\right)=3 X^{4}+12 X^{3}+42 X^{2}+48 X+120$ in positive integers $X$ and $Y$ with $X=x_{1}+1 \geq 2$. Observing that $(X, Y)=$ $(1,15)$ is an integral point on this curve, we find by using the MAGMA command

$$
\text { IntegralQuarticPoints([3, 12, 42, 48, 120], [1, 15]); }
$$

that all integral points on the curve are given by

$$
(X, Y) \in\{(1, \pm 15),(-2, \pm 12),(-14, \pm 300),(-29, \pm 1365)\} .
$$


Since none of the points $(X, Y)$ satisfy $X \geq 2$, we exclude the case $a_{2}=2$, $a_{4}=6$. Further, when $a_{2}=2, a_{5}=5$, by putting $X=x_{1}+1$ and $Y=$ $10 x_{2} x_{5}$, we get the curve $Y^{2}=10\left(X^{2}+4\right)\left((X+3)^{2}+4\right)=10 X^{4}+60 X^{3}+$ $170 X^{2}+240 X+520$ on which $(X, Y)=(-1,20)$ is an integral point. It follows by MAGMA that all the integral points on the curve satisfy $X \leq 1$, and also this case is excluded. When $a_{4}=6, a_{5}=5$, by putting $X=x_{1}+3$ and $Y=30 x_{4} x_{5}$, we get the curve $Y^{2}=30\left(X^{2}+6\right)\left((X+1)^{2}+4\right)=$ $30 X^{4}+60 X^{3}+330 X^{2}+360 X+900$ on which $(X, Y)=(0,30)$ is an integral point. It follows by MAGMA that all the integral points on the curve other than $(X, Y)=(11,500)$ satisfy $X \leq 1$. Since $X>1,30 \mid Y$ and $30 \nmid 500$, also this case is excluded. When (2.5) holds, we get $5 d=2 x_{1}+1$, and this with $n+d=x_{1}^{2}$ implies

$$
\begin{array}{ll}
2\left(5 x_{2}\right)^{2}=25(n+d)+25 d=25 x_{1}^{2}+10 x_{1}+5=\left(5 x_{1}+1\right)^{2}+4 & \text { if } a_{2}=2, \\
6\left(5 x_{4}\right)^{2}=25(n+d)+75 d=25 x_{1}^{2}+30 x_{1}+15=\left(5 x_{1}+3\right)^{2}+6 & \text { if } a_{4}=6, \\
5\left(5 x_{5}\right)^{2}=25(n+d)+100 d=25 x_{1}^{2}+40 x_{1}+20=\left(5 x_{1}+4\right)^{2}+4 & \text { if } a_{5}=5 .
\end{array}
$$

As in the case (2.4), these give rise to the same quartic curves $Y^{2}=3 X^{4}+$ $12 X^{3}+42 X^{2}+48 X+120 ; Y^{2}=10 X^{4}+60 X^{3}+170 X^{2}+240 X+520$; and $Y^{2}=30 X^{4}+60 X^{3}+330 X^{2}+360 X+900$ when $a_{2}=2, a_{3}=6 ; a_{2}=2$, $a_{5}=5$; and $a_{4}=6, a_{5}=5$, respectively. This is not possible.

Similarly all the other cases are excluded. In case (iii), we have $n=2 x_{0}^{2}$ and obtain either $d=2 x_{0}+3$ or $3 d=2 x_{0}+1$. Then we use $2 a_{i} x_{i}^{2}=$ $2(n+i d)=\left(2 x_{0}\right)^{2}+2 i\left(2 x_{0}+3\right)=\left(2 x_{0}+i\right)^{2}+6 i-i^{2}$ if $d=2 x_{0}+3$ and $2 a_{i}\left(3 x_{i}\right)^{2}=18(n+i d)=\left(6 x_{0}\right)^{2}+6 i\left(2 x_{0}+1\right)=\left(6 x_{0}+i\right)^{2}+6 i-i^{2}$ if $3 d=2 x_{0}+1$ to get quartic equations. In case (vi), we obtain the quartic equation $Y^{2}=6 X^{4}+36 X^{3}+108 X-54=6\left(X^{4}+6 X^{3}+18 X-9\right)$. For any integral point $(X, Y)$ on this curve, we obtain $3 \mid\left(X^{4}+6 X^{3}+18 X-9\right)$, giving $3 \mid X$. Then $\operatorname{ord}_{3}\left(X^{4}+6 X^{3}+18 X-9\right)=2$, giving $\operatorname{ord}_{3}\left(Y^{2}\right)=\operatorname{ord}_{3}(6)+2=3$, a contradiction.

3. Proof of Lemma 2.5. For the proof of Lemma 2.5, we use the so-called elliptic Chabauty method (see [NB02], [NB03]). Bruin's routines related to the elliptic Chabauty method are contained in [MAGMA], so here we indicate the main steps only, and a MAGMA routine which can be used to verify the computations is available from the third author.

First consider the tuple $(6,7,2,1,10, *, 3)$. Using the equalities $n=$ $-2(n+3 d)+3(n+2 d)=-2 x_{3}^{2}+6 x_{2}^{2}$ and $d=(n+3 d)-(n+2 d)=x_{3}^{2}-2 x_{2}^{2}$ we obtain the following system of equations:

$$
\begin{aligned}
-x_{3}^{2}+3 x_{2}^{2} & =3 x_{0}^{2}, \\
-x_{3}^{2}+4 x_{2}^{2} & =7 x_{1}^{2}, \quad 4 x_{3}^{2}-6 x_{2}^{2}=5 x_{4}^{2},
\end{aligned}
$$


The first equation implies that $x_{3}$ is divisible by 3 , that is, there exists a $z \in \mathbb{Z}$ such that $x_{3}=3 z$. By standard factorization argument we get

$$
\left(\sqrt{3} z+x_{2}\right)\left(3 z+x_{2}\right)\left(12 z^{2}-2 x_{2}^{2}\right)=\delta \square,
$$

where $\delta \in\{ \pm 2+\sqrt{3}, \pm 10+5 \sqrt{3}\}$. Thus putting $X=z / x_{2}$ it is sufficient to find all points $(X, Y)$ on the curves

$$
C_{\delta}: \quad \delta(\sqrt{3} X+1)(3 X+1)\left(12 X^{2}-2\right)=Y^{2},
$$

for which $X \in \mathbb{Q}$ and $Y \in \mathbb{Q}(\sqrt{3})$. For all possible values of $\delta$ the point $(X, Y)=(-1 / 3,0)$ is on the curves, therefore we can transform them to elliptic curves. We note that $X=z / x_{2}=-1 / 3$ does not yield appropriate arithmetic progressions.

I. $\delta=2+\sqrt{3}$. In this case $C_{2+\sqrt{3}}$ is isomorphic to the elliptic curve

$$
E_{2+\sqrt{3}}: \quad y^{2}=x^{3}+(-\sqrt{3}-1) x^{2}+(6 \sqrt{3}-9) x+(11 \sqrt{3}-19) .
$$

Using MAGMA, we find that the rank of $E_{2+\sqrt{3}}$ is 0 and the only point on $C_{2+\sqrt{3}}$ for which $X \in \mathbb{Q}$ is $(X, Y)=(-1 / 3,0)$.

II. $\delta=-2+\sqrt{3}$. Applying elliptic Chabauty with $p=7$, we deduce that $z / x_{2} \in\{-1 / 2,-1 / 3,-33 / 74,0\}$. Among these values, $z / x_{2}=-1 / 2$ gives $n=6, d=1$.

III. $\delta=10+5 \sqrt{3}$. Applying again elliptic Chabauty with $p=23$ shows that $z / x_{2} \in\{1 / 2,-1 / 3\}$. Here $z / x_{2}=1 / 2$ corresponds to $n=6, d=1$.

IV. $\delta=-10+5 \sqrt{3}$. The elliptic curve $E_{-10+5 \sqrt{3}}$ is of rank 0 and the only point on $C_{-10+5 \sqrt{3}}$ for which $X \in \mathbb{Q}$ is $(X, Y)=(-1 / 3,0)$.

We have proved that there is no arithmetic progression with $\left(a_{0}, a_{1}, \ldots\right.$ $\left.\ldots, a_{6}\right)=(6,7,2,1,10, *, 3)$ and $d \nmid n$.

Now consider the tuple $(1,5,6,7,2, *, 10)$. The system of equations we use is

$$
\begin{aligned}
x_{6}^{2}-3 x_{1}^{2} & =-2\left(x_{0} / 2\right)^{2}, & 4 x_{6}^{2}+3 x_{1}^{2} & =7 x_{3}^{2}, \\
x_{6}^{2}+2 x_{1}^{2} & =3 x_{2}^{2}, & 3 x_{6}^{2}+x_{1}^{2} & =x_{4}^{2} .
\end{aligned}
$$

We factor the first equation over $\mathbb{Q}(\sqrt{3})$ and the fourth over $\mathbb{Q}(\sqrt{-3})$. We obtain

where

$$
x_{6}+\sqrt{3} x_{1}=\delta_{1} \square, \quad \frac{\sqrt{-3} x_{6}+x_{1}}{2}=\delta_{2} \square,
$$

$$
\begin{aligned}
& \delta_{1} \in\{ \pm 1+\sqrt{3}, \pm 1-\sqrt{3}, \pm 5+3 \sqrt{3}, \pm 5-3 \sqrt{3}\} \\
& \delta_{2} \in\{ \pm 1,( \pm 1+\sqrt{-3}) / 2,( \pm 1-\sqrt{-3}) / 2\} .
\end{aligned}
$$

The curves for which we apply the elliptic Chabauty method are

$$
C_{\delta}: \quad 3 \delta(X+\sqrt{3})(\sqrt{-3} X+1)\left(X^{2}+2\right)=Y^{2},
$$


defined over $\mathbb{Q}(\alpha)$, where $\alpha^{4}+36=0$. It turns out that there is no arithmetic progression with $\left(a_{0}, a_{1}, \ldots, a_{6}\right)=(1,5,6,7,2, *, 10)$ and $d \nmid n$.

We now make some observations. If

$$
u(n+i d)+v(n+j d)=w(n+l d)
$$

holds with $0 \leq i, j, l \leq k-1$ and integers $u, v, w$, then

$$
u+v=w \quad \text { and } \quad u i+v j=w l .
$$

Therefore

$$
u(n+(k-1-i) d)+v(n+(k-1-j) d)=w(n+(k-1-l) d)
$$

holds, and vice versa. Therefore any tuple $\left(a_{0}, a_{1}, \ldots, a_{6}\right)$ and its mirror tuple $\left(a_{6}, \ldots, a_{1}, a_{0}\right)$ give rise to the same set of equations. Hence it suffices to exclude any one of them. Also it suffices to exclude any one of $\left(*, a_{1}, \ldots, a_{6}\right)$ and $\left(a_{0}, a_{1}, \ldots, a_{5}, *\right)$.

Further, if we define $a_{i}^{\prime}=a_{i} / 2$ if $a_{i}$ is even and $a_{i}^{\prime}=2 a_{i}$ if $a_{i}$ is odd, then $\left(a_{0}^{\prime}, a_{1}^{\prime}, \ldots, a_{6}^{\prime}\right)$ and $\left(a_{0}, a_{1}, \ldots, a_{6}\right)$ give rise to the same set of equations. Let $i, j, l$ satisfy (3.2). If $n+i d=a_{i} x_{i}^{2}, n+j d=a_{j} x_{j}^{2}, n+l d=a_{l} x_{l}^{2}$ is the one given by $\left(a_{0}, a_{1}, \ldots, a_{6}\right)$, and $n+i d=a_{i}^{\prime} x_{i}^{\prime 2}, n+j d=a_{j}^{\prime} x_{j}^{\prime 2}, n+l d=a_{l}^{\prime} x_{l}^{\prime 2}$ the one given by $\left(a_{0}^{\prime}, a_{1}^{\prime}, \ldots, a_{6}^{\prime}\right)$, then from (3.2) we get

$$
u a_{i} x_{i}^{2}+v a_{j} x_{j}^{2}=w a_{l} x_{l}^{2}
$$

and

$$
u a_{i}^{\prime} x_{i}^{\prime 2}+v a_{j}^{\prime} x_{j}^{\prime 2}=w a_{l}^{\prime} x_{l}^{\prime 2}
$$

respectively. Since $2 a_{i}^{\prime} x_{i}^{\prime 2}=a_{i} y_{i}^{2}$ for some $y_{i}$, multiplying (3.4) by 2 , we obtain an equation exactly similar to (3.3). Hence if we exclude one of $\left(a_{0}^{\prime}, a_{1}^{\prime}, \ldots, a_{6}^{\prime}\right)$ or $\left(a_{0}, a_{1}, \ldots, a_{6}\right)$, the other tuple is excluded.

In view of the above observations and since $\left(a_{0}, a_{1}, \ldots, a_{6}\right)=(1,2,3, *, 5$, $6,7)$ is excluded if $\left(a_{1}, a_{2}, \ldots, a_{6}\right)=(1,2,3, *, 5,6)$ is excluded, it suffices to consider the tuples

$$
\begin{aligned}
\left(a_{0}, a_{1}, \ldots, a_{6}\right) \in\{ & (*, 3,1,5,6,7,2),(3, *, 5,6,7,2,1),(1,5,6,7,2, *, 10), \\
& (*, 5,6,7,2,1,10),(6,7,2,1,10, *, 3),(*, 1,2,3, *, 5,6)\} .
\end{aligned}
$$

Already the tuples $\left(a_{0}, a_{1}, \ldots, a_{6}\right) \in\{(1,5,6,7,2, *, 10),(6,7,2,1,10, *, 3)\}$ are excluded. In the table below, we indicate the relevant quartic polynomials for the remaining tuples:

\begin{tabular}{ll}
\hline Tuple & Polynomial \\
\hline$(1,2,3, *, 5,6)$ & $2 \delta_{A 1}(X+\sqrt{-1})(X+3 \sqrt{-1})\left(5 X^{2}-3\right)$ \\
$(*, 3,1,5,6,7,2)$ & $\delta_{A 2}(X+\sqrt{-1})(2 X+\sqrt{-1})\left(5 X^{2}-1\right)$ \\
$(3, *, 5,6,7,2,1)$ & $5 \delta_{A 3}(2 X+3 \sqrt{-1})(X+\sqrt{-1})\left(12 X^{2}-3\right)$ \\
$(*, 5,6,7,2,1,10)$ & $\delta_{A 4}(X+\sqrt{-2})(2 \sqrt{-2} X+1)\left(3 X^{2}+1\right)$ \\
\hline
\end{tabular}


4. Proof of Theorem 1. Suppose that the assumptions of Theorem 1 are satisfied and assume (1.1) with $\omega(d)=1$. Let $k \geq 9$. By Lemma 2.3, we may suppose that $P(b)=k$, implying $k$ is a prime. After deleting the term divisible by $k$ on the left hand side of (1.1) and using Lemma 2.4, the assertion for $k \geq 15$ follows. Thus it suffices to prove the assertion for $k \in\{7,8,11,13\}$ with $P(b) \leq k$ for $k \in\{7,8\}$ and $P(b)=k$ for $k \in\{11,13\}$. Therefore we always restrict to $k \in\{7,8,11,13\}$ and $P(b) \leq k$ for $k \in$ $\{7,8\}$ and $P(b)=k$ for $k \in\{11,13\}$. In view of Lemma 2.1, we arrive at a contradiction by showing $t-|R| \geq 2$ when $k \in\{11,13\}$. Further, Lemma 2.1 also implies that $p \nmid d$ for $p \leq k$ whenever $k \in\{11,13\}$.

For a prime $p \leq k$ and $p \nmid d$, let $i_{p}$ be such that $0 \leq i_{p}<p$ and $p \mid n+i_{p} d$. For any subset $\mathcal{I} \subseteq[0, k) \cap \mathbb{Z}$ and primes $p_{1}, p_{2}$ with $p_{i} \leq k$ and $p_{i} \nmid d$, $i=1,2$, we define

$$
\begin{aligned}
& \mathcal{I}_{1}=\left\{i \in \mathcal{I}:\left(\frac{i-i_{p_{1}}}{p_{1}}\right)=\left(\frac{i-i_{p_{2}}}{p_{2}}\right)\right\}, \\
& \mathcal{I}_{2}=\left\{i \in \mathcal{I}:\left(\frac{i-i_{p_{1}}}{p_{1}}\right) \neq\left(\frac{i-i_{p_{2}}}{p_{2}}\right)\right\} .
\end{aligned}
$$

Then from $\left(\frac{a_{i}}{p}\right)=\left(\frac{i-i_{p}}{p}\right)\left(\frac{d}{p}\right)$, we see that either

$$
\left(\frac{a_{i}}{p_{1}}\right) \neq\left(\frac{a_{i}}{p_{2}}\right) \text { for all } i \in \mathcal{I}_{1} \quad \text { and } \quad\left(\frac{a_{i}}{p_{1}}\right)=\left(\frac{a_{i}}{p_{2}}\right) \text { for all } i \in \mathcal{I}_{2} \text {, }
$$

or

$$
\left(\frac{a_{i}}{p_{1}}\right) \neq\left(\frac{a_{i}}{p_{2}}\right) \text { for all } i \in \mathcal{I}_{2} \quad \text { and } \quad\left(\frac{a_{i}}{p_{1}}\right)=\left(\frac{a_{i}}{p_{2}}\right) \text { for all } i \in \mathcal{I}_{1} .
$$

We define $(\mathcal{M}, \mathcal{B})=\left(\mathcal{I}_{1}, \mathcal{I}_{2}\right)$ in the case $(4.1)$ and $(\mathcal{M}, \mathcal{B})=\left(\mathcal{I}_{2}, \mathcal{I}_{1}\right)$ in the case (4.2). We write $\left(\mathcal{I}_{1}, \mathcal{I}_{2}, \mathcal{M}, \mathcal{B}\right)=\left(\mathcal{I}_{1}^{k}, \mathcal{I}_{2}^{k}, \mathcal{M}^{k}, \mathcal{B}^{k}\right)$ when $\mathcal{I}=[0, k) \cap \mathbb{Z}$. Then for any $\mathcal{I} \subseteq[0, k) \cap \mathbb{Z}$, we have

$$
\mathcal{I}_{1} \subseteq \mathcal{I}_{1}^{k}, \quad \mathcal{I}_{2} \subseteq \mathcal{I}_{2}^{k}, \quad \mathcal{M} \subseteq \mathcal{M}^{k}, \quad \mathcal{B} \subseteq \mathcal{B}^{k}
$$

and

$$
|\mathcal{M}| \geq\left|\mathcal{M}^{k}\right|-(k-|\mathcal{I}|), \quad|\mathcal{B}| \geq\left|\mathcal{B}^{k}\right|-(k-|\mathcal{I}|) .
$$

By taking $m=n+\gamma_{t} d$ and $\gamma_{i}^{\prime}=\gamma_{t}-\gamma_{t-i+1}$, we rewrite (1.1) as

$$
\left(m-\gamma_{1}^{\prime} d\right) \cdots\left(m-\gamma_{t}^{\prime} d\right)=b y^{2} .
$$

The equation (4.4) is called the mirror image of (1.1). The corresponding $t$-tuple $\left(a_{\gamma_{1}^{\prime}}, \ldots, a_{\gamma_{t}^{\prime}}\right)$ is called the mirror image of $\left(a_{\gamma_{1}}, \ldots, a_{\gamma_{t}}\right)$.

4.1. The case $k=7,8$. We may assume that $k=7$ since the case $k=8$ follows from that of $k=7$.

In this subsection, we take $d \in\left\{2^{\alpha}, p^{\alpha}, 2 p^{\alpha}\right\}$ where $p$ is any odd prime and $\alpha$ is a positive integer. In fact, we prove 
Lemma 4.1. Let $\psi=1, k=7$ and $d \nmid n$. Then (1.1) with $d \in\left\{2^{\alpha}, p^{\alpha}, 2 p^{\alpha}\right\}$ does not hold.

First we check that (1.1) does not hold for $d \leq 23$ and $n+5 d \leq 324$. Thus we assume that either $d>23$ or $n+5 d>324$. Hence $n+i d>24 i$, since $n>208$ if $d \leq 23$. Then (1.1) with $\psi=0, k \geq 4$ and $\omega(d)=1$ has no solution by Lemma 2.2. Let $d=2$ or $d=4$. Suppose $a_{i}=a_{j}$ with $i>j$. Then $x_{i}-x_{j}=r_{1}$ and $x_{i}+x_{j}=r_{2}$ with $r_{1}, r_{2}$ even and $\operatorname{gcd}\left(r_{1}, r_{2}\right)=2$. Now from $a_{i} x_{i}^{2}=n+i d>24 i \geq 4 i^{2}$, we get

$$
i-j \geq \frac{a_{i}\left(x_{i}+x_{j}\right)}{2} \geq \frac{\left(a_{i} x_{i}^{2}\right)^{1 / 2}+\left(a_{j} x_{j}^{2}\right)^{1 / 2}}{2}>\frac{2 i+2 j}{2} \geq i,
$$

a contradiction. Therefore $a_{i} \neq a_{j}$ whenever $i \neq j$, giving $|R|=k-1$. But $\left|\left\{a_{i}: P\left(a_{i}\right) \leq 5\right\}\right| \leq 4$, implying $|R| \leq 4+1<k-1$, a contradiction. Let $8 \mid d$. From $(2.1)$, we get $\left(\frac{a_{i}}{8}\right)=\left(\frac{n+i d}{8}\right)=\left(\frac{n}{8}\right)$, implying $a_{i}$ 's belong each to exactly one distinct residue class modulo 8 . Therefore $\left|\left\{a_{i}: P\left(a_{i}\right) \leq 5\right\}\right| \leq 1$, which together with $\left|\left\{j: a_{j}=a_{i}\right\}\right| \leq 2$ for $a_{i} \in R$ implies $\left|\left\{i: P\left(a_{i}\right) \leq 5\right\}\right| \leq 2$. This is a contradiction since $\left|\left\{i: P\left(a_{i}\right) \leq 5\right\}\right| \geq 7-2=5$. Thus $d \neq 2^{\alpha}$. Let $t-|R| \geq 2$. Then we observe from [LaSh07, Corollary 3.10] that $d_{2}=d<24$ and $n+5 d<324$. This is not possible.

Therefore $t-|R| \leq 1$, implying $|R| \geq k-2=5$. If $7 \mid d$, then we get a contradiction since $7 \nmid a_{i}$ for any $i$ and $\left|\left\{a_{i}: P\left(a_{i}\right) \leq 5\right\}\right| \leq 4$, implying $|R| \leq 4<k-2$. If $3 \mid d$ or $5 \mid d$, then we also obtain a contradiction since $\left|\left\{a_{i}: P\left(a_{i}\right) \leq 5\right\}\right| \leq 2$, implying $|R| \leq 2+1<k-2$.

Thus $\operatorname{gcd}(p, d)=1$ for each prime $p \leq 7$. Therefore $5 \mid n+i_{5} d$ and $7 \mid n+i_{7} d$ with $0 \leq i_{5}<5$ and $0 \leq i_{7}<7$. By taking the mirror image (4.4) of (1.1), we may suppose that $0 \leq i_{7} \leq 3$.

Let $p_{1}=5, p_{2}=7$ and $\mathcal{I}=\left\{\gamma_{1}, \ldots, \gamma_{6}\right\}$. We observe that $P\left(a_{i}\right) \leq 3$ for $i \in \mathcal{M} \cup \mathcal{B}$. Since $\left(\frac{2}{5}\right) \neq\left(\frac{2}{7}\right)$ but $\left(\frac{3}{5}\right)=\left(\frac{3}{7}\right)$, we observe that $a_{i} \in\{2,6\}$ whenever $i \in \mathcal{M}$ and $a_{i} \in\{1,3\}$ whenever $i \in \mathcal{B}$.

We now define four sets

$$
\begin{aligned}
& \mathcal{I}_{++}^{k}=\left\{i: 0 \leq i<k,\left(\frac{i-i_{p_{1}}}{p_{1}}\right)=\left(\frac{i-i_{p_{2}}}{p_{2}}\right)=1\right\}, \\
& \mathcal{I}_{--}^{k}=\left\{i: 0 \leq i<k,\left(\frac{i-i_{p_{1}}}{p_{1}}\right)=\left(\frac{i-i_{p_{2}}}{p_{2}}\right)=-1\right\}, \\
& \mathcal{I}_{+-}^{k}=\left\{i: 0 \leq i<k,\left(\frac{i-i_{p_{1}}}{p_{1}}\right)=1,\left(\frac{i-i_{p_{2}}}{p_{2}}\right)=-1\right\}, \\
& \mathcal{I}_{-+}^{k}=\left\{i: 0 \leq i<k,\left(\frac{i-i_{p_{1}}}{p_{1}}\right)=-1,\left(\frac{i-i_{p_{2}}}{p_{2}}\right)=1\right\}
\end{aligned}
$$

and let $\mathcal{I}_{++}=\mathcal{I}_{++}^{k} \cap \mathcal{I}, \mathcal{I}_{--}=\mathcal{I}_{--}^{k} \cap \mathcal{I}, \mathcal{I}_{+-}=\mathcal{I}_{+-}^{k} \cap \mathcal{I}, \mathcal{I}_{-+}=\mathcal{I}_{-+}^{k} \cap \mathcal{I}$. We observe that $\mathcal{I}_{1}=\mathcal{I}_{++} \cup \mathcal{I}_{--}$and $\mathcal{I}_{2}=\mathcal{I}_{+-} \cup \mathcal{I}_{-+}$. Since $a_{i} \in\{1,2,3,6\}$ 
for $i \in \mathcal{I}_{1} \cup \mathcal{I}_{2}$ and $\left(\frac{a_{i}}{p}\right)=\left(\frac{i-i_{p}}{p}\right)\left(\frac{d}{p}\right)$, we obtain four possibilities $I, I I, I I I$ and $I V$ according as $\left(\frac{d}{5}\right)=\left(\frac{d}{7}\right)=1 ;\left(\frac{d}{5}\right)=\left(\frac{d}{7}\right)=-1 ;\left(\frac{d}{5}\right)=1,\left(\frac{d}{7}\right)=-1$; $\left(\frac{d}{5}\right)=-1,\left(\frac{d}{7}\right)=1$, respectively.

\begin{tabular}{ccccc}
\hline & $\left\{a_{i}: i \in \mathcal{I}_{++}\right\}$ & $\left\{a_{i}: i \in \mathcal{I}_{--}\right\}$ & $\left\{a_{i}: i \in \mathcal{I}_{+-}\right\}$ & $\left\{a_{i}: i \in \mathcal{I}_{-+}\right\}$ \\
\hline$I$ & $\{1\}$ & $\{3\}$ & $\{6\}$ & $\{2\}$ \\
$I I$ & $\{3\}$ & $\{1\}$ & $\{2\}$ & $\{6\}$ \\
$I I I$ & $\{2\}$ & $\{6\}$ & $\{3\}$ & $\{1\}$ \\
$I V$ & $\{6\}$ & $\{2\}$ & $\{1\}$ & $\{3\}$ \\
\hline
\end{tabular}

In case $I$, we have $\left(\frac{a_{i}}{p}\right)=\left(\frac{i-i_{p}}{p}\right)$ for $p \in\{5,7\}$, which together with $\left(\frac{a_{i}}{5}\right)=1$ for $a_{i} \in\{1,6\},\left(\frac{a_{i}}{5}\right)=-1$ for $a_{i} \in\{2,3\},\left(\frac{a_{i}}{7}\right)=1$ for $a_{i} \in\{1,2\}$ and $\left(\frac{a_{i}}{7}\right)$ $=-1$ for $a_{i} \in\{3,6\}$ implies the assertion. The assertion for cases II, III and $I V$ follows similarly. For simplicity, we write $\mathcal{A}_{7}=\left(a_{0}, a_{1}, a_{2}, a_{3}, a_{4}, a_{5}, a_{6}\right)$.

For each possibility $0 \leq i_{5}<5$ and $0 \leq i_{7} \leq 3$, we compute $\mathcal{I}_{++}^{k}$, $\mathcal{I}_{--}^{k}, \mathcal{I}_{+-}^{k}, \mathcal{I}_{-+}^{k}$ and restrict to those pairs $\left(i_{5}, i_{7}\right)$ for which $\max \left(\left|\mathcal{I}_{1}^{k}\right|,\left|\mathcal{I}_{2}^{k}\right|\right)$ $\leq 4$. Then we check for the possibilities $I, I I, I I I$ or $I V$.

Suppose $d=2 p^{\alpha}$. Then $b_{i} \in\{1,3\}$ whenever $P\left(b_{i}\right) \leq 3$. If $i_{5} \neq 0,1$, then $|R| \leq 2+2=4$, giving $t-|R| \geq 7-1-4=2$, a contradiction. Thus $i_{5} \in\{0,1\}$. Further, $\mathcal{M}=\emptyset$ and $a_{i} \in\{1,3\}$ for $i \in \mathcal{B}$. Therefore either $\left|\mathcal{I}_{1}^{k}\right| \leq 1$ or $\left|\mathcal{I}_{1}^{k}\right| \leq 2$. We find that this is the case only when $\left(i_{5}, i_{7}\right) \in$ $\{(0,1),(1,2)\}$. Let $\left(i_{5}, i_{7}\right)=(0,1)$. We get $\mathcal{I}_{++}^{k}=\mathcal{I}_{--}^{k}=\emptyset, \mathcal{I}_{+-}^{k}=\{4,6\}$ and $\mathcal{I}_{-+}^{k}=\{2,3\}$. It suffices to consider cases $I I I$ and $I V$, since $b_{i} \in\{1,3\}$ whenever $P\left(b_{i}\right) \leq 3$. Suppose $I I I$ holds. Then by reducing modulo 3 , we obtain $4 \notin \mathcal{I}, a_{6}=3$ and $a_{2}=a_{3}=1$. By reducing modulo 3 again, we get $a_{1} \notin\{1,7,3\}$ which is not possible since $5 \nmid a_{1}$. Suppose $I V$ holds. Then by reducing modulo 3 , we obtain $2 \notin \mathcal{I}, a_{4}=a_{6}=1$ and $a_{3}=3$. We now get $a_{1} \in\{1,7\}$ and as $t-|R| \leq 1$, we get $a_{1}=7$. This is not possible since $-1=\left(\frac{a_{1} a_{4}}{5}\right)=\left(\frac{(1-0)(4-0)}{5}\right)=1$. Similarly $\left(i_{5}, i_{7}\right)=(1,2)$ is excluded. Hence $d=p^{\alpha}$ from now on.

Let $\left(i_{5}, i_{7}\right)=(0,0)$. We obtain $\mathcal{I}_{++}^{k}=\{1,4\}, \mathcal{I}_{--}^{k}=\{3\}, \mathcal{I}_{+-}^{k}=\{6\}$ and $\mathcal{I}_{-+}^{k}=\{2\}$. We may assume that $1 \in \mathcal{I}$, as otherwise $P\left(a_{2} a_{3} a_{4} a_{5} a_{6}\right) \leq 5$ and this is excluded by Lemma 2.2 with $k=5$. Further, $i \notin \mathcal{I}$ for exactly one of $i \in\{2,3,4\}$, as otherwise $P\left(a_{1} a_{2} a_{3} a_{4}\right) \leq 3$ and this is not possible by Lemma 2.2 with $k=4$ since $d>23$. Consider the possibilities $I I$ and $I V$. By reducing modulo 3 , we obtain $2 \notin \mathcal{I}, 3 \mid a_{1} a_{4}$ and $a_{3} a_{6}=2$. This is not possible modulo 3 since $-1=\left(\frac{a_{3} a_{6}}{3}\right)=\left(\frac{(3-1)(6-1)}{3}\right)=1$, a contradiction. Suppose $I$ holds. Then $a_{1}=1$ and $a_{6}=6$. If $4 \in \mathcal{I}$, then $a_{1}=a_{4}=1$ and at least one of $a_{3}=3, a_{2}=2$ holds, which is excluded by Lemma 2.7(i). Assume that $4 \notin \mathcal{I}$. Then $a_{1}=1, a_{2}=2, a_{3}=3, a_{6}=6$, giving $a_{5}=5$ by reducing modulo 2 and 3 . Thus we have $\left(a_{1}, \ldots, a_{5}, a_{6}\right)=(1,2,3, *, 5,6)$. 
This is not possible by Lemma 2.5. Suppose $I I I$ holds. Then $4 \notin \mathcal{I}, a_{1}=2$, $a_{2}=1, a_{3}=6, a_{6}=3$, giving $a_{5}=10$ by reducing modulo 2 and 3 . Thus $\left(a_{1}, \ldots, a_{5}, a_{6}\right)=(2,1,6, *, 10,3)$ which is also excluded by Lemma 2.5.

Let $\left(i_{5}, i_{7}\right)=(0,1)$. We obtain $\mathcal{I}_{++}^{k}=\mathcal{I}_{--}^{k}=\emptyset, \mathcal{I}_{+-}^{k}=\{4,6\}$ and $\mathcal{I}_{-+}^{k}=\{2,3\}$. The possibility $I$ is excluded by parity and modulo 3 . The possibility $I I$ implies that $3 \notin \mathcal{I}, a_{4}=a_{6}=2$ and $a_{2}=3$. This is not possible modulo 3 . Suppose $I I I$ holds. Then $a_{2}=a_{3}=1$ and either $4 \notin \mathcal{I}$, $a_{6}=3$ or $6 \notin \mathcal{I}, a_{4}=3$. By reducing modulo 3 , we obtain $4 \notin \mathcal{I}, a_{6}=3$ and $\left(\frac{a_{5}}{3}\right)=\left(\frac{a_{2}}{3}\right)=1$. This gives $a_{5} \in\{1,10\}$, which together with $t-|R| \leq 1$ implies $a_{5}=10$. But this is not possible by Lemma 2.6 with $n^{\prime}=n+2 d$, $d^{\prime}=d$ and $(i, j, l)=(1,3,4)$. Hence $I I I$ is excluded. Suppose $I V$ holds. Then $a_{4}=a_{6}=1$ and $2 \notin \mathcal{I}, a_{3}=3$ by reducing modulo 3 . By reducting modulo 3 , we get $a_{5} \in\{2,5\}$ and we may take $a_{5}=5$, as otherwise we get a contradiction from $d>23$ and Lemma 2.2 with $k=4$ applied to $(n+3 d)(n+4 d)(n+5 d)(n+6 d)$. This is again not possible by Lemma 2.6 with $n^{\prime}=n+3 d, d^{\prime}=d$ and $(i, j, l)=(1,2,3)$.

Let $\left(i_{5}, i_{7}\right)=(0,3)$. We obtain $\mathcal{I}_{++}^{k}=\{4\}, \mathcal{I}_{--}^{k}=\{2\}, \mathcal{I}_{+-}^{k}=\{1,6\}$ and $\mathcal{I}_{-+}^{k}=\emptyset$. By reducing modulo 3 , we observe that the possibilities $I$ and $I I I$ are excluded. Suppose $I I$ happens. Then $a_{2}=1, a_{4}=3$ and either $a_{6}=2$, $1 \notin \mathcal{I}$ or $a_{1}=2,6 \notin \mathcal{I}$. If $a_{6}=2,1 \notin \mathcal{I}$, then $a_{5} \in\{1,5\}$, which gives $a_{5}=1$ by reducing modulo 3 . This is not possible modulo 7 since $-1=\left(\frac{a_{4} a_{5}}{7}\right)=$ $\left(\frac{(4-3)(5-3)}{7}\right)=1$. Thus $a_{1}=2,6 \notin \mathcal{I}$. Then $a_{0}=5, a_{5}=10, a_{3}=14$ by reducing modulo 3 , giving $\left(a_{0}, a_{1}, \ldots, a_{5}, a_{6}\right)=(5,2,1,14,3,10, *)$. Suppose $I V$ happens. Let $1,6 \in \mathcal{I}$. Then $a_{1}=a_{6}=1$ and either $a_{2}=2$ or $a_{4}=6$. By Lemma 2.7(ii), we may assume that either $2 \notin \mathcal{I}$ or $4 \notin \mathcal{I}$. If $2 \notin \mathcal{I}$, then $a_{4}=6, a_{3}=7$ and $a_{5}=5$, which is excluded by Lemma 2.7(ii). Thus $4 \notin \mathcal{I}, a_{2}=2$ and $a_{5}=5$ since $3 \nmid a_{5}$. This is also excluded by Lemma 2.7(ii). Therefore $a_{2}=2, a_{4}=6$ and either $6 \notin \mathcal{I}, a_{1}=1$ or $1 \notin \mathcal{I}, a_{6}=1$. Now $7 \mid a_{3}$, as otherwise $P\left(a_{1} a_{2} \ldots a_{5}\right) \leq 5$ if $1 \in \mathcal{I}$ or $P\left(a_{2} a_{3} \ldots a_{6}\right) \leq 5$ if $6 \in \mathcal{I}$, and this is excluded by Lemma 2.2 with $k=5$. Further, by reducing modulo 3 , we get $a_{3}=7, a_{0}=10$ and $a_{5}=5$. Hence we obtain $\mathcal{A}_{7}=(10, *, 2,7,6,5,1)$ or $\mathcal{A}_{7}=(10,1,2,7,6,5, *)$.

Let $\left(i_{5}, i_{7}\right)=(1,0)$. We obtain $\mathcal{I}_{++}^{k}=\{2\}, \mathcal{I}_{--}^{k}=\{3\}, \mathcal{I}_{+-}^{k}=\{5\}$ and $\mathcal{I}_{-+}^{k}=\{4\}$. We consider the possibility $I$. By a parity argument, we have either $5 \notin \mathcal{I}$ or $4 \notin \mathcal{I}$. Again by reducing modulo 3 , either $3 \notin \mathcal{I}$ or $5 \notin \mathcal{I}$. Thus $5 \notin \mathcal{I}$, giving $a_{2}=1, a_{3}=3, a_{4}=2$. Now $5 \mid a_{1}$, as otherwise we get a contradiction from $P\left(a_{1} a_{2} a_{3} a_{4}\right) \leq 3$, Lemma 2.2 with $k=4$ and $d>23$. Hence $a_{1}=5$. This is again a contradiction since $-1=\left(\frac{a_{1} a_{2}}{7}\right)=$ $\left(\frac{(1-0)(2-0)}{7}\right)=1$. Thus the possibility $I$ is excluded. If $I I I$ holds, then $3 \notin \mathcal{I}$, $a_{2}=2, a_{5}=3, a_{4}=1$, giving $a_{1} \in\{1,5\}$ and $a_{6}=5$. By reducing modulo 3 , we get $a_{1}=1$. But this is not possible by Lemma 2.6 with $n^{\prime}=n+2 d$, 
$d^{\prime}=d$ and $(i, j, l)=(1,3,4)$. Similarly, the possibilities $I I$ and $I V$ are also excluded. If $I I$ holds, then $4 \notin \mathcal{I}, a_{2}=3, a_{3}=1, a_{5}=2$. Now $a_{6} \in\{1,5\}$ and by further reducing modulo 3 , we get $a_{6}=1$. This is not possible by Lemma 2.6 with $n^{\prime}=n+2 d, d^{\prime}=d$ and $(i, j, l)=(1,3,4)$. If $I V$ holds, then $2 \notin \mathcal{I}, a_{3}=2, a_{5}=1, a_{4}=3$. Then $a_{6} \in\{1,5\}$, giving $a_{6}=5$ by reducing modulo 3 . This is not possible modulo 7 .

Let $\left(i_{5}, i_{7}\right)=(1,1)$. We obtain $\mathcal{I}_{++}^{k}=\{2,5\}, \mathcal{I}_{--}^{k}=\{4\}, \mathcal{I}_{+-}^{k}=\{0\}$ and $\mathcal{I}_{-+}^{k}=\{3\}$. We consider the possibilities $I I I$ and $I V$. By parity, we obtain $5 \notin \mathcal{I}$. But then we get a contradiction modulo 3 since $a_{4}=6$, $a_{0}=3$ if $I I I$ holds and $a_{2}=6, a_{3}=3$ if $I V$ holds are not possible. Next we consider the possibility $I$. Then $0 \notin \mathcal{I}$ by reducing modulo 2 and 3 and we get $P\left(a_{2} a_{3} \ldots a_{6}\right) \leq 5$, which is excluded by Lemma 2.2 with $k=5$. Let $I I$ hold. Then $3 \notin \mathcal{I}$ by reducing modulo 2 and 3 and $a_{2}=a_{5}=3, a_{4}=1, a_{0}=2$. Further, $a_{6} \in\{5,10\}$ which together with reduction modulo 3 gives $a_{6}=5$. Now we get a contradiction modulo 7 from $a_{5}=3, a_{6}=5$.

Let $\left(i_{5}, i_{7}\right)=(3,1)$. We obtain $\mathcal{I}_{++}^{k}=\{2\}, \mathcal{I}_{--}^{k}=\{0,6\}, \mathcal{I}_{+-}^{k}=\{4\}$ and $\mathcal{I}_{-+}^{k}=\{5\}$. We may assume that $i \notin \mathcal{I}$ for exactly one of $i \in\{0,2,4,6\}$, as otherwise $n$ is even, $P\left(a_{0} a_{2} a_{4} a_{6}\right) \leq 3$ and this is excluded by $k=4$ of Lemma 2.2 applied to $(n / 2)(n / 2+d)(n / 2+2 d)(n / 2+3 d)$. We consider the possibilities $I$ and $I I I$. By reducing modulo 3 , we get $4 \notin \mathcal{I}, a_{0}=a_{6}, 3 \mid a_{0}$ and $a_{2} a_{5}=2$. This is not possible by reducing modulo 3 . Next we consider the possibility $I I$. Then $4 \notin \mathcal{I}$ by a parity argument. Further, $a_{0}=a_{6}=1$, $a_{2}=3, a_{5}=6$. This is not possible since $8 \mid x_{6}^{2}-x_{0}^{2}=n+6 d-n=6 d$ and $d$ is odd. Finally, we consider the possibility $I V$. If $2 \notin \mathcal{I}$ or $4 \notin \mathcal{I}$, then $a_{0}=a_{6}=2, a_{5}=3$ and one of $a_{2}=6$ and $a_{4}=1$. This is excluded by Lemma 2.7(iii). Thus $a_{2}=6, a_{4}=1, a_{5}=3$ and either $a_{0}=2,6 \notin \mathcal{I}$ or $a_{6}=2,0 \notin \mathcal{I}$. Then $a_{1}=7, a_{3}=5$ by parity and reduction modulo 3 . Hence $\mathcal{A}_{7}=(2,7,6,5,1,3, *)$ or $\mathcal{A}_{7}=(*, 7,6,5,1,3,2)$.

All the other pairs are excluded similarly. For $\left(i_{5}, i_{7}\right)=(0,2)$, we obtain either $\mathcal{A}_{7}=(1,2,3, *, 5,6,7)$ or $(5,6,7,2,1,10, *)$ or $(10,3,14,1,2,5, *)$, which are all excluded by Lemma 2.5 . For $\left(i_{5}, i_{7}\right)=(1,3)$, we obtain $\mathcal{A}_{7}=$ $(1,5,6,7,2, *, 10),(*, 5,6,7,2,1,10)$ or $(*, 10,3,14,1,2,5)$, which is not possible by Lemma 2.5 , or $a_{0}=a_{5}=1$ and at least two of $a_{1}=5, a_{2}=6$, $a_{4}=2$ hold, which is again excluded by Lemma $2.7(\mathrm{iv})$. For $\left(i_{5}, i_{7}\right)=(2,0)$, we obtain $\mathcal{A}_{7}=(14,3,10, *, 6,1,2),(7,6,5, *, 3,2,1)$ or $a_{3}=a_{6}=1, a_{0}=7$, $a_{1}=6, a_{2}=5, a_{4}=3$ or $a_{5}=2$. These are impossible by Lemma $2.7(\mathrm{v})$. For $\left(i_{5}, i_{7}\right)=(2,1)$, we obtain $a_{0}=a_{4}=1, a_{3}=3, a_{6}=2$, which is not possible by Lemma $2.7(\mathrm{vi})$. For $\left(i_{5}, i_{7}\right)=(4,1)$, we obtain $\mathcal{A}_{7}=$ $(6,7,2,1,10, *, 3)$, which is also excluded. For $\left(i_{5}, i_{7}\right)=(4,2)$, we obtain $\mathcal{A}_{7}=(2,1,14,3,10, *, 6),(1,2,7,6,5, *, 3),(*, 2,7,6,5,1,3)$ or $a_{0}=a_{5}=1$ and at least two of $a_{1}=2, a_{3}=6, a_{6}=3$ hold. The previous possibility is excluded by Lemma 2.5 and the latter by Lemma 2.7 (vii). 
4.2. The case $k=11$. We may assume that $11 \mid a_{i}$ for some $i \in\{4,5,6\}$ whenever $i \notin \mathcal{I}$, as otherwise the lemma follows from Lemma 4.1.

Let $p_{1}=5, p_{2}=11$ and $\mathcal{I}=\left\{\gamma_{1}, \ldots, \gamma_{t}\right\}$. We observe that $P\left(a_{i}\right) \leq 7$ for $i \in \mathcal{M} \cup \mathcal{B}$. Since $\left(\frac{3}{5}\right) \neq\left(\frac{3}{11}\right)$ but $\left(\frac{q}{5}\right)=\left(\frac{q}{11}\right)$ for a prime $q<k$ other than $3,5,11$, we observe that $3 \mid a_{i}$ whenever $i \in \mathcal{M}$. Since $\sigma_{3} \leq 4$ and $|\mathcal{I}|=k-1$, we deduce from (4.3) that $\left|\mathcal{M}^{k}\right| \leq 5$ and $3 \mid a_{i}$ for at least $\left|\mathcal{M}^{k}\right|-1$ elements $i \in \mathcal{M}^{k}$. Further, $a_{i} \in\{1,2,7,14\}$ for $i \in \mathcal{B}$, giving $|\mathcal{B}| \leq 5$, as otherwise $t-|R| \geq 2$. Hence $\left|\mathcal{B}^{k}\right| \leq 6$ by (4.3).

By taking the mirror image (4.4) of (1.1), we may suppose that $4 \leq i_{11}$ $\leq 5$. For each possibility $0 \leq i_{5}<5$ and $4 \leq i_{11} \leq 5$, we compute $\left|\mathcal{I}_{1}^{k}\right|,\left|\mathcal{I}_{2}^{k}\right|$ and restrict to those pairs $\left(i_{5}, i_{11}\right)$ for which $\max \left(\left|\mathcal{I}_{1}^{k}\right|,\left|\mathcal{I}_{2}^{k}\right|\right) \leq 6$. Further, we restrict to those pairs $\left(i_{5}, i_{11}\right)$ for which either

$$
3 \mid a_{i} \text { for at least }\left|\mathcal{I}_{1}^{k}\right|-1 \text { elements } i \in \mathcal{I}_{1}^{k},
$$

or

$$
3 \mid a_{i} \text { for at least }\left|\mathcal{I}_{2}^{k}\right|-1 \text { elements } i \in \mathcal{I}_{2}^{k} .
$$

We find that exactly one of (4.5) or (4.6) happens. We have $\mathcal{M}^{k}=\mathcal{I}_{1}^{k}$, $\mathcal{B}^{k}=\mathcal{I}_{2}^{k}$ when (4.5) holds, and $\mathcal{M}^{k}=\mathcal{I}_{2}^{k}, \mathcal{B}^{k}=\mathcal{I}_{1}^{k}$ when (4.6) holds. If $3 \mid a_{i}$ for exactly $\left|\mathcal{M}^{k}\right|-1$ elements $i \in \mathcal{M}^{k}$, then $\mathcal{B}=\mathcal{B}^{k}$ and we restrict to such pairs $\left(i_{5}, i_{11}\right)$ for which there are at most three elements $i \in \mathcal{B}^{k}$ with $P\left(a_{i}\right) \leq 2$, as otherwise $t-|R| \geq 2$. Now all the pairs $\left(i_{5}, i_{11}\right)$ are excluded other than

$$
(0,4),(1,5),(4,5) \text {. }
$$

For these pairs, we find that $\left|\mathcal{B}^{k}\right| \geq 5$. Hence we may suppose that $7 \mid a_{i}$ for some $i \in \mathcal{B}$, as otherwise $a_{i} \in\{1,2\}$ for $i \in \mathcal{B}$, which together with $|\mathcal{B}| \geq 4$ gives $t-|R| \geq 2$. Further, if $\left|\mathcal{B}^{k}\right|=6$, then we may assume that $7\left|a_{i}, 7\right| a_{i+7}$ for some $0 \leq i \leq 3$.

Let $\left(i_{5}, i_{11}\right)=(0,4)$. Then $\mathcal{M}^{k}=\{3,9\}$ and $\mathcal{B}^{k}=\{1,2,6,7,8\}$, giving $i_{3}=0$. If $7 \mid a_{6} a_{7}$, then $|\mathcal{B}|=\left|\mathcal{B}^{k}\right|-1$ and $a_{i} \in\{3,6\}$ for $i \in \mathcal{M}=\mathcal{M}^{k}$ but $\left(\frac{a_{3} a_{9}}{7}\right)=\left(\frac{\left(3-i_{7}\right)\left(9-i_{7}\right)}{7}\right)=-1$ for $i_{7}=6,7$, a contradiction. If $7 \mid a_{2}$, then $a_{i} \in\{5,10\}$ for $i \in\{5,10\} \subseteq \mathcal{I}$ but $\left(\frac{a_{5} a_{10}}{7}\right)=\left(\frac{(5-2)(10-2)}{7}\right)=-1$, a contradiction again. Thus $7 \mid a_{1} a_{8}$ and $a_{i} \in\{1,2\}$ for $\{2,6,7\} \cap \mathcal{B}^{k}$. From $\left(\frac{a_{i}}{7}\right)=\left(\frac{i-1}{7}\right)\left(\frac{d}{7}\right),\left(\frac{6-1}{7}\right)=\left(\frac{7-1}{7}\right)=-1$ and $\left(\frac{2-1}{7}\right)=1$, we find that $2 \notin \mathcal{I}$. This is not possible.

Let $\left(i_{5}, i_{11}\right)=(1,5)$. Then $\mathcal{M}^{k}=\{4,10\}$ and $\mathcal{B}^{k}=\{0,2,3,7,8,9\}$, giving $i_{3}=1$. Thus $\mathcal{M}=\mathcal{M}^{k}, a_{i} \in\{3,6\}$ for $i \in \mathcal{M}$ and $|\mathcal{B}|=\left|\mathcal{B}^{k}\right|-1$, $a_{i} \in\{1,2,7,14\}$ for $i \in \mathcal{B}$. Further, we have either $7 \mid a_{0} a_{7}$ or $7 \mid a_{2} a_{9}$. Taking $\left(\frac{a_{i}}{7}\right)$ for $i \in\{4,10,0,2,3,7,8,9\}$, we find that $7 \mid a_{2} a_{9}$ and $3 \notin \mathcal{B}$. This is not possible. 
Let $\left(i_{5}, i_{11}\right)=(4,5)$. Then $\mathcal{M}^{k}=\{0,6\}$ and $\mathcal{B}^{k}=\{1,2,3,7,8,10\}$, giving $\mathcal{M}=\mathcal{M}^{k}$ and $i_{3}=0$. Further, $7 \mid a_{1} a_{8}$ or $7 \mid a_{3} a_{10}$. Taking $\left(\frac{a_{i}}{7}\right)$ for $i \in \mathcal{M} \cup \mathcal{B}^{k}$, we find that $7 \mid a_{1} a_{8}$ and $\mathcal{B}=\mathcal{B}^{k} \backslash\{7\}$. This is not possible since $7 \in \mathcal{I}$.

4.3. The case $k=13$. We may assume that $13 \mid a_{3} a_{4} a_{5} a_{6} a_{7} a_{8} a_{9}$, otherwise the assertion follows from Theorem 1 with $k=11$.

Let $p_{1}=11, p_{2}=13$ and $\mathcal{I}=\left\{\gamma_{1}, \ldots, \gamma_{t}\right\}$. Since $\left(\frac{5}{11}\right) \neq\left(\frac{5}{13}\right)$ but $\left(\frac{q}{11}\right)=\left(\frac{q}{13}\right)$ for $q=2,3,7$, we observe that for $5 \mid a_{i}$ for $i \in \mathcal{M}$ and $P\left(a_{i}\right) \leq 7$, $5 \nmid a_{i}$ for $i \in \mathcal{B}$. Since $\sigma_{5} \leq 3$, we obtain $\left|\mathcal{M}^{k}\right| \leq 4$ and $5 \mid a_{i}$ for at least $\left|\mathcal{M}^{k}\right|-1$ elements $i \in \mathcal{M}^{k}$.

By taking the mirror image (4.4) of (1.1), we may suppose that $3 \leq i_{13}$ $\leq 6$ and $0 \leq i_{11} \leq 10$. We may suppose that $i_{13} \geq 4,5$ if $i_{11}=0,1$ respectively, and $\max \left(i_{11}, i_{13}\right) \geq 6$ if $i_{11} \geq 2$, as otherwise the assertion follows from Lemma 4.1.

Since $\max \left(\left|\mathcal{I}_{1}^{k}\right|,\left|\mathcal{I}_{2}^{k}\right|\right) \geq 5$ and $\left|\mathcal{M}^{k}\right| \leq 4$, we restrict to those pairs satisfying $\min \left(\left|\mathcal{I}_{1}^{k}\right|,\left|\mathcal{I}_{2}^{k}\right|\right) \leq 4$, and further $\mathcal{M}^{k}$ is exactly one of $\mathcal{I}_{1}^{k}$ or $\mathcal{I}_{2}^{k}$ with minimum cardinality and hence $\mathcal{B}^{k}$ is the other one. Now we restrict to those pairs $\left(i_{11}, i_{13}\right)$ for which $5 \mid a_{i}$ for at least $\left|\mathcal{M}^{k}\right|-1$ elements $i \in \mathcal{M}^{k}$. If $5 \mid a_{i}$ for exactly $\left|\mathcal{M}^{k}\right|-1$ elements $i \in \mathcal{M}^{k}$, then $\mathcal{B}=\mathcal{B}^{k}$ and hence we may assume that $|\mathcal{B}|=\left|\mathcal{B}^{k}\right| \leq 7$, as otherwise there are at least six elements $i \in \mathcal{B}$ for which $a_{i} \in\{1,2,3,6\}$, giving $t-|R| \geq 2$. Therefore we now exclude those pairs $\left(i_{11}, i_{13}\right)$ for which $5 \mid a_{i}$ for exactly $\left|\mathcal{M}^{k}\right|-1$ elements $i \in \mathcal{M}^{k}$ and $\left|\mathcal{B}^{k}\right|>7$. We find that all the pairs $\left(i_{11}, i_{13}\right)$ are excluded other than

$$
(1,3),(2,4),(3,5),(4,2),(5,3),(6,4) \text {. }
$$

From $i_{13} \geq 5$ if $i_{11}=1$ and $\max \left(i_{11}, i_{13}\right) \geq 6$ if $i_{11} \geq 2$, we find that all these pairs are excluded other than $(6,4)$.

Let $\left(i_{11}, i_{13}\right)=(6,4)$. Then $\mathcal{M}^{k}=\{0,2,7,12\}$ and $\mathcal{B}^{k}=\{1,3,5,8,9$, $10,11\}$, giving $i_{5}=1, \mathcal{M}=\{2,7,12\}$ and $0 \notin \mathcal{I}$. This is excluded by applying Lemma 4.1 to $\prod_{i=0}^{5}(n+d+2 i)$.

5. Proof of Theorem 2. By Lemma 2.2, we may suppose that $P(b)>k$. If $P(b)=p_{\pi(k)+1}$ or $P(b)=p_{\pi(k)+2}$ with $p_{\pi(k)+1} \nmid b$, then the assertion follows from Theorem 1. Thus we may suppose that $P(b)=p_{\pi(k)+2}$ and $p_{\pi(k)+1} \mid b$. Then we delete the terms divisible by $p_{\pi(k)+1}, p_{\pi(k)+2}$ on the left hand side of (1.1), and the assertion for $k \geq 15$ follows from Lemma 2.4. Thus $11 \leq k \leq 14$ and it suffices to prove the assertion for $k=11$ and $k=13$. After removing the $i$ 's for which $p \mid a_{i}$ with $p \in\{13,17\}$ when $k=11$ and $p \mid a_{i}$ with $p \in\{17,19\}$ when $k=13$, we observe from Lemma 2.1 that $k-|R| \leq 1$ and $p \nmid d$ for each $p \leq k$. 
5.1. The case $k=11$. Let $p_{1}=11, p_{2}=13$ and $\mathcal{I}=\{0,1, \ldots, 10\}$. Since $\left(\frac{5}{11}\right) \neq\left(\frac{5}{13}\right),\left(\frac{17}{11}\right) \neq\left(\frac{17}{13}\right)$ but $\left(\frac{q}{11}\right)=\left(\frac{q}{13}\right)$ for $q=2,3,7$, we observe that either $5 \mid a_{i}$ or $17 \mid a_{i}$ for $i \in \mathcal{M}$ and either $5 \cdot 17 \mid a_{i}$ or $P\left(a_{i}\right) \leq 7$ for $i \in \mathcal{B}$. Since $\sigma_{5} \leq 3$, we obtain $|\mathcal{M}| \leq 4$.

By taking the mirror image (4.4) of (1.1), we may suppose that $0 \leq$ $i_{13} \leq 5$ and $0 \leq i_{11} \leq 10$. If both $i_{11}, i_{13}$ are odd, then we may suppose that $i_{17}$ is even, as otherwise we get a contradiction from Lemma 4.1 applied to $\prod_{i=0}^{5}(n+i(2 d))$. Also we may suppose that $\max \left(i_{11}, i_{13}\right) \geq 4$, as otherwise we get a contradiction from Lemma 4.1 applied to $\prod_{i=0}^{6}(n+4 d+i d)$. Further, from Lemma 4.1, we may assume $i_{17}>4$ if $\max \left(i_{11}, i_{13}\right)=4$.

Since $\max \left(\left|\mathcal{I}_{1}^{k}\right|,\left|\mathcal{I}_{2}^{k}\right|\right) \geq 5$ and $\left|\mathcal{M}^{k}\right| \leq 4$, we restrict to those pairs satisfying $\min \left(\left|\mathcal{I}_{1}^{k}\right|,\left|\mathcal{I}_{2}^{k}\right|\right) \leq 4$, and further $\mathcal{M}^{k}$ is exactly one of $\mathcal{I}_{1}^{k}$ or $\mathcal{I}_{2}^{k}$ with minimum cardinality and hence $\mathcal{B}^{k}$ is the other one. Now we restrict to those pairs $\left(i_{11}, i_{13}\right)$ for which either $5 \mid a_{i}$ or $17 \mid a_{i}$ whenever $i \in \mathcal{M}$. Let $\mathcal{B}^{\prime}=\mathcal{B} \backslash\left\{i: 5 \cdot 17 \mid a_{i}\right\}$. If $\left|\mathcal{B}^{\prime}\right| \geq 8$, then there are at least six elements $i \in \mathcal{B}^{\prime}$ such that $P\left(a_{i}\right) \leq 3$, giving $k-|R| \geq 2$. Thus we restrict to those pairs for which $\left|\mathcal{B}^{\prime}\right| \leq 7$. Further, we observe that $7 \mid a_{i}$ and $7 \mid a_{i+7}$ for some $i, i+7 \in \mathcal{B}^{\prime}$ if $\left|\mathcal{B}^{\prime}\right|=7$.

Let $\left(i_{11}, i_{13}\right)=(2,4)$. Then $\mathcal{M}^{k}=\{1,6,8\}$ and $\mathcal{B}^{k}=\{0,3,5,7,9,10\}$, giving $i_{5}=1,17 \mid a_{8}$ and $P\left(a_{i}\right) \leq 7$ for $i \in \mathcal{B}$. For each possibility $i_{7} \in$ $\{0,3,4,5\}$, and $i_{17}=8$, we take $p_{1}=7, p_{2}=17, \mathcal{I}=\mathcal{B}^{k}$ and compute $\mathcal{I}_{1}$ and $\mathcal{I}_{2}$. Since $\left(\frac{p}{7}\right)=\left(\frac{p}{17}\right)$ for $p \in\{2,3\}$, we should have either $\mathcal{I}_{1}=\emptyset$ or $\mathcal{I}_{2}=\emptyset$. We find that $\min \left(\left|\mathcal{I}_{1}\right|,\left|\mathcal{I}_{2}\right|\right)>0$ for each possibility $i_{7} \in\{0,3,4,5\}$. Hence $\left(i_{11}, i_{13}\right)=(2,4)$ is excluded. Similarly all pairs $\left(i_{11}, i_{13}\right)$ are excluded except $\left(i_{11}, i_{13}\right) \in\{(4,2),(6,4)\}$. When $\left(i_{11}, i_{13}\right)=(3,5)$, we get $\mathcal{M}^{k}=$ $\{2,7,9\}$, giving $5\left|a_{2} a_{7}, 17\right| a_{9}$ and hence it is excluded. When $\left(i_{11}, i_{13}\right)=$ $(1,4)$, we obtain $\mathcal{M}^{k}=\{5,9\}$ and $\mathcal{B}^{k}=\{0,2,3,6,7,8,10\}$, giving either $5\left|a_{5}, 17\right| a_{9}$ or $17\left|a_{5}, 5\right| a_{9}$. Also $i_{7} \in\{0,3\}$. Thus we have $\left(i_{7}, i_{17}\right) \in\{(0,5)$, $(0,9),(3,5),(3,9)\}$ and apply the procedure for each of these possibilities.

Let $\left(i_{11}, i_{13}\right)=(6,4)$. Then $\mathcal{M}^{k}=\{0,2,7\}$ and $\mathcal{B}^{k}=\{1,3,5,8,9,10\}$, giving $i_{5}=2,17 \mid a_{0}$ and $P\left(a_{i}\right) \leq 7$ for $i \in \mathcal{B}$. For each possibility $i_{7} \in$ $\{1,3,4,5\}$, and $i_{17}=0$, we take $p_{1}=7, p_{2}=17$ and $\mathcal{I}=\mathcal{B}^{k}$. Since $\left(\frac{p}{7}\right)=$ $\left(\frac{p}{17}\right)$ for $p \in\{2,3\}$, we observe that either $\mathcal{I}_{1}=\emptyset$ or $\mathcal{I}_{2}=\emptyset$. We find that this happens only when $i_{7}=3$ where we get $\mathcal{I}_{1}=\emptyset$ and $\mathcal{I}_{2}=\{1,5,8,9\}$. By reducing modulo 7 , we get $a_{i} \in\{1,2\}$ for $i \in\{1,8,9\}$ and $a_{5} \in\{3,6\}$. Further, by reducing modulo 5 , we obtain $a_{1}=a_{8}=1, a_{9}=2, a_{5}=3$, $a_{1}=4, a_{10}=7$, and this is excluded by Runge's method as in [MuSh03]. When $\left(i_{11}, i_{13}\right)=(4,2)$, we get $\mathcal{M}^{k}=\{0,5,10\}$ and $\mathcal{B}^{k}=\{1,3,6,7,8,9\}$, giving $5 \mid a_{0} a_{5} a_{10}$ and $i_{17} \in\{5,10\}$. Here we obtain $i_{17}=10, i_{7}=3$ where $\mathcal{I}_{1}=\emptyset$ and $\mathcal{I}_{2}=\{1,6,7,8,9\}$. This is not possible by Lemma 2.2 with $k=4$ applied to $(n+6 d)(n+6 d+d)(n+6 d+2 d)(n+6 d+3 d)$. 
5.2. The case $k=13$. Let $p_{1}=11, p_{2}=13$ and $\mathcal{I}=\{0,1, \ldots, 12\}$. Since $\left(\frac{5}{11}\right) \neq\left(\frac{5}{13}\right),\left(\frac{17}{11}\right) \neq\left(\frac{17}{13}\right)$ but $\left(\frac{q}{11}\right)=\left(\frac{q}{13}\right)$ for $q=2,3,7$, we observe that either $5 \mid a_{i}$ or $17 \mid a_{i}$ for $i \in \mathcal{M}^{k}$ and either $5 \cdot 17 \mid a_{i}$ or $19 \mid a_{i}$ or $P\left(a_{i}\right) \leq 7$ for $i \in \mathcal{B}^{k}$. Since $\sigma_{5} \leq 3$, we obtain $\left|\mathcal{M}^{k}\right| \leq 4$.

By taking the mirror image (4.4) of (1.1), we may suppose that $0 \leq i_{13}$ $\leq 6$ and $0 \leq i_{11} \leq 10$. We may assume that $i_{11}, i_{13}, i_{17}, i_{19}$ are not all even, as otherwise $P\left(\prod_{i=0}^{5} a_{2 i+1}\right) \leq 7$, which is excluded by Lemma 4.1. Further, exactly two of $i_{11}, i_{13}, i_{17}, i_{19}$ are even and the other two odd, as otherwise this is excluded again by Lemma 4.1 applied to $\prod_{i=0}^{6}(n+i(2 d))$ if $n$ is odd and $\prod_{i=0}^{6}(n / 2+i d)$ if $n$ is even. Also exactly two of $i_{11}, i_{13}, i_{17}, i_{19}$ lie in each set $\{2,3,4,5,6,7,8\}$ and $\{3,4,5,6,7,8,9\}$, otherwise this is excluded by Lemma 4.1 .

Since $\max \left(\left|\mathcal{I}_{1}^{k}\right|,\left|\mathcal{I}_{2}^{k}\right|\right) \geq 5$ and $\left|\mathcal{M}^{k}\right| \leq 4$, we restrict to those pairs satisfying $\min \left(\left|\mathcal{I}_{1}^{k}\right|,\left|\mathcal{I}_{2}^{k}\right|\right) \leq 4$, and further $\mathcal{M}^{k}$ is exactly one of $\mathcal{I}_{1}^{k}$ or $\mathcal{I}_{2}^{k}$ with minimum cardinality and hence $\mathcal{B}^{k}$ is the other one. Now we restrict to those pairs $\left(i_{11}, i_{13}\right)$ for which either $5 \mid a_{i}$ or $17 \mid a_{i}$ whenever $i \in \mathcal{M}$. Let $\mathcal{B}^{\prime}=\mathcal{B}^{k} \backslash\left\{i: 5 \cdot 17 \mid a_{i}\right\}$. If $\left|\mathcal{B}^{\prime}\right| \geq 9$, then there are at least six elements $i \in \mathcal{B}^{\prime}$ such that $P\left(a_{i}\right) \leq 3$, giving $k-|R| \geq 2$. Thus we restrict to those pairs for which $\left|\mathcal{B}^{\prime}\right| \leq 8$. For instance, let $\left(i_{11}, i_{13}\right)=(0,0)$. We obtain $\mathcal{M}^{k}=\{5,10\}$ and $\mathcal{B}^{k}=\{1,2,3,4,6,7,8,9,12\}$, giving $i_{5}=0, i_{17} \in\{5,10\}, \mathcal{B}^{\prime}=\mathcal{B}^{k}$ and $\left|\mathcal{B}^{k}\right|=9$. This is excluded.

Let $\left(i_{11}, i_{13}\right)=(1,1)$. Then $\mathcal{M}^{k}=\{0,6,11\}$ and $\mathcal{B}^{k}=\{2,3,4,5,7,8,9,10\}$, giving $i_{5}=1, i_{17}=0$. This is excluded. Similarly $\left(i_{11}, i_{13}\right) \in\{(1,3),(2,4)$, $(3,5),(4,6),(6,4),(7,5),(8,6)\}$ are excluded where we find that $i_{17}$ is of the same parity as $i_{11}, i_{13}$.

Let $\left(i_{11}, i_{13}\right)=(4,2)$. Then $\mathcal{M}^{k}=\{0,5,10\}$ and $\mathcal{B}^{k}=\{1,3,6,7,8,9$, $11,12\}$, giving $5\left|a_{0}, 5\right| a_{10}$ and $i_{17}=5$. Further, for $i \in \mathcal{B}^{k}$, we have either $19 \mid a_{i}$ or $P\left(a_{i}\right) \leq 7$. Also $7 \mid a_{1}$ and $7 \mid a_{8}$, as otherwise $k-|R| \geq 2$. We now take $\left(i_{7}, i_{17}\right)=(1,5), p_{1}=7, p_{2}=17, \mathcal{I}=\mathcal{B}^{k}$ and compute $\mathcal{I}_{1}$ and $\mathcal{I}_{2}$. Since $\left(\frac{p}{7}\right)=\left(\frac{p}{17}\right)$ for $p \in\{2,3\}$, and $\left(\frac{19}{7}\right)=\left(\frac{19}{17}\right)$, we should have either $\left|\mathcal{I}_{1}\right|=1$ or $\left|\mathcal{I}_{2}\right|=1$. We find that $\mathcal{I}_{1}=\{3,9,11\}, \mathcal{I}_{2}=\{6,7,12\}$, which is a contradiction. Similarly $\left(i_{11}, i_{13}\right) \in\{(5,3),(8,4)\}$ are also excluded. When $\left(i_{11}, i_{13}\right)=(5,3)$, we find that $i_{17}=6$ and $i_{7} \in\{0,2\}$, and this is excluded.

6. A remark. We consider (1.1) with $\psi=0, \omega(d)=2$ and the assumption $\operatorname{gcd}(n, d)=1$ replaced by $d \nmid n$ if $b>1$. It is proved in [LaSh07] that (1.1) with $\psi=0, b=1$ and $k \geq 8$ is not possible. We show that (1.1) with $\psi=0, k \geq 6$ and $\omega(d)=2$ is not possible. The case $k=6$ has already been solved in [BBGH06]. Let $k \geq 7$. As in [LaSh07] and since $d \nmid n$, the assertion follows if (1.1) with $\psi=1, k \geq 7, \omega(d)=1$ and $\operatorname{gcd}(n, d)=1$ does not hold. This follows from Theorem 1. 


\section{References}

[BBGH06] M. A. Bennett, N. Bruin, K. Győry and L. Hajdu, Powers from products of consecutive terms in arithmetic progression, Proc. London Math. Soc. 92 (2006), 273-306.

[MAGMA] W. Bosma, J. Cannon and C. Playoust, The Magma algebra system. I. The user language, J. Symbolic Comput. 24 (1997), 235-265.

[NB02] N. Bruin, Chabauty Methods and Covering Techniques Applied to Generalized Fermat Equations, CWI Tract 133, Stichting Math. Centrum, Centrum voor Wiskunde en Informatica, Amsterdam, 2002.

[NB03] - Chabauty methods using elliptic curves, J. Reine Angew. Math. 562 (2003), 27-49.

[LaSh07] S. Laishram and T. N. Shorey, The equation $n(n+d) \cdots(n+(k-1) d)=b y^{2}$ with $\omega(d) \leq 6$ or $d \leq 10^{10}$, Acta Arith. 129 (2007), 249-305.

[LaSh08] - - - Squares in arithmetic progression with at most two terms omitted, ibid. 134 (2008), 299-316.

[MuSh03] A. Mukhopadhyay and T. N. Shorey, Almost squares in arithmetic progression (II), ibid. 110 (2003), 1-14.

[MuSh04] -, -, Almost squares in arithmetic progression (III), Indag. Math. 15 (2004), 523-533.

[SaSh03] N. Saradha and T. N. Shorey, Almost squares in arithmetic progression, Compos. Math. 138 (2003), 73-111.

[TSH06] T. N. Shorey, Powers in arithmetic progression (III), in: The Riemann Zeta Function and Related Themes, Ramanujan Math. Soc. Publ., 2006, 131-140.

[Sz08] Sz. Tengely, Note on a paper "An extension of a theorem of Euler" by HirataKohno et al. (Acta Arith. 129 (2007), 71-102), Acta Arith. 134 (2008), 329335.

Department of Pure Mathematics

University of Waterloo

200 University Avenue West

Waterloo, ON, N2L 3G1, Canada

E-mail:shanta@math.tifr.res.in

Institute of Mathematics

University of Debrecen

and the Number Theory Research Group

of the Hungarian Academy of Sciences

P.O. Box 12, 4010 Debrecen, Hungary

E-mail: tengely@math.klte.hu
School of Mathematics Tata Institute of Fundamental Research Homi Bhabha Road Mumbai 400 005, India E-mail: shorey@math.tifr.res.in

Received on 19.12.2007

and in revised form on 4.8.2008 